\title{
Isolation and Identification of Leishmania Species From Sandflies and Rodents Collected From Roffaye District, Khuzestan Province, Southwest of Iran
}

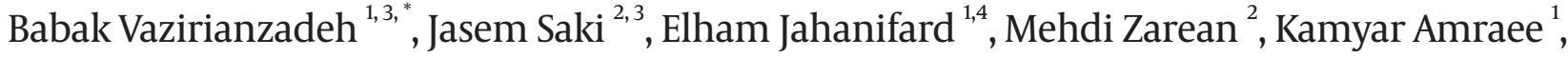 \\ Sharokh Navid Pour ${ }^{5}$ \\ ${ }_{2}^{1}$ Department of Medical Entomology, Ahvaz Jundishapur University Medical Sciences, Ahvaz, IR Iran \\ 2 Department of Medical Parasitology, Ahvaz Jundishapur University Medical Sciences, Ahvaz, IR Iran \\ ${ }_{4}^{3}$ Infectious and Tropical Diseases Research Center, Ahvaz Jundishapur University Medical Sciences, Ahvaz, IR Iran \\ 4 Department of Medical Entomology and Vector Control, School of Public Health, Tehran University Medical Sciences, Tehran, IR Iran \\ ${ }^{5}$ Razi Vaccine and Serum Research Institute, Karaj, IR Iran \\ ${ }^{*}$ Corresponding author: Babak Vazirianzadeh, Department of Medical Entomology and Infectious and Tropical Diseases Research Centre, Ahvaz Jundishapur University Medical Sci- \\ ences, Ahvaz, IR Iran. Tel/Fax: +98-9163095110, E-mail: Babakvazir@yahoo.co.uk.
}

Received: December 29, 2012; Revised: May 04, 2013; Accepted: May 11, 2013

\begin{abstract}
Background: Leishmaniais which is one of the six most important tropical diseases in the world are in Iran in 3 forms. Cutaneous leishmaniasis (CL) is endemic in Iran, however,Zoonotic Cutaneous Leishmaniasis (ZCL) is endemic in Khuzestan province.

Objectives: This study aimed to determine the species of Leishmania in vector and reservoir hosts of cutaneous leishmaniasis based on molecular methods in Roffaye District, Khuzestan, Iran.

Materials and Methods: In this study sand flies were collected from indoors and outdoors using sticky traps and rodents using Sherman live traps. They were both subjected to the nested PCR method to detect Leishmania parasite.

Results: Phlebotomus papatasi was the most common species of sandflies in outdoor and indoor resting places and Tatera indica (Indian gerbil) was the most common species among rodents. PCR technique showed that only 2 out of the 27 P.papatasi (7.4\%) and 1 of the Tatera indica out of 12 rodents (8.3\%) were positive for parasite due Leishmania major. The trapped Alactaga sp. were not infected by L. major.

Conclusions: This is the first molecular report on parasite infection of both vector (P.papatas) and reservoir(T.indica) to L. major from this area. Therefore, the population of both sandflies and rodents should be reduced using control methods.
\end{abstract}

Keywords: Leishmaniasis; Nested PCR; Phlebotomus papatasi; Tatera indica

\section{Background}

Leishmaniasis is among the six most important tropical diseases while its different aspects are recommended as research topics by the World Health Organization $(1,2)$. Three forms of leishmaniasis including: Zoonotic Cutaneous Leishmaniasis (ZCL), Anthroponotic Cutaneous Leishmaniasis (ACL) and Visceral Leishmaniasis (VL) have been causing some health and medical problems related to humans and animals in Iran and its adjoining countries such as Iraq, Afganistan and Pakistan (3, 4). Cutaneous Leishmaniasis (CL) is considered as an important health problem in almost all countries of the Middle East including Iran $(1,2)$.

Cutaneous leishmaniasis is endemic in two forms ACL and ZCL, however ZCL is endemic in the Khuzestan prov- ince and caused by the zooflagellate parasite Leishmania major $(5,6)$. The main vector of this parasite in Iran, including Khuzestan is female Phlebotomus papatasi Scopoli (Diptera: Psychodidae). Tatera indica, the Indian gerbil, is the main reservoir host of $L$. major in the southwest of Iran $(2,5)$. Estimate shave shown an increase in the rate of ZCL cases in different regions of Khuzestan including western districts among the human population during the passed decade (7-9).

\section{Objectives}

One of these areas was Roffaye near the border with Iraq. Therefore, the present study was carried out to detect the nature of this parasite among trapped sandflies and rodent reservoir.

Implication for health policy/practice/research/medical education

Molecular detection of Leishmania species in the vector and reservoir at same time is important in determining both the control method and treatment of Cutaneous Leishmaniasis.

Copyright (C) 2013, Ahvaz Jundishapur University of Medical Sciences; Licensee Kowsar Ltd. This is an Open Access article distributed under the terms of the Creative Commons Attribution License (http://creativecommons.org/licenses/by/3.0), which permits unrestricted use, distribution, and reproduction in any medium, provided the original work is properly cited. 


\section{Materials and Methods}

\subsection{Study Area}

Dasht-e-Azadegan County is located in the north-west of Ahvaz and has a border with Iraq. Latitude and longitude of this county are $31^{\circ} 33^{\prime} \mathrm{N}$ and $48^{\circ} 10^{\prime} \mathrm{E}$, respectively. Altitude from sea level is $10 \mathrm{~m}$. It includes 3 regions; central, Hoveyzeh and Boustan. Hoveyzeh region has 2 cities; Rofayyeh and Hoveyzeh. Rofayyeh ( $31^{\circ} 55^{\prime} \mathrm{N}, 47^{\circ} 40^{\prime}$ ), the area of this research study is situated $25 \mathrm{~km}$ from the west of Hoveyzeh and $50 \mathrm{~km}$ from the southwest of Susangerd city, the center of Dasht-e-Azadegan, between the Iran and Iraq border (Figure 1).

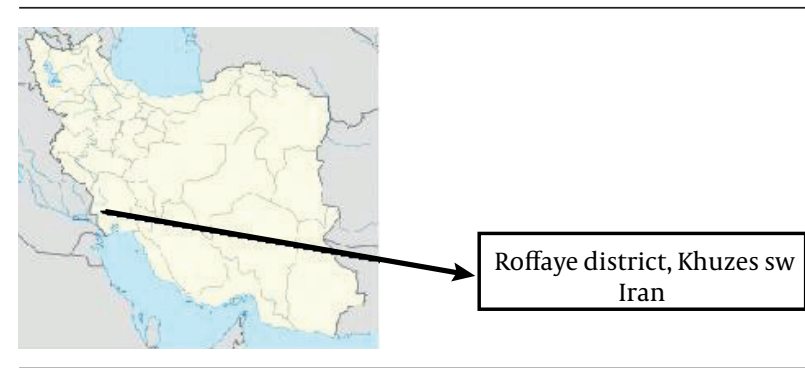

Figure 1. Roffaye district, Khuzestan, South-West Iran $\left(31^{\circ} 35^{\prime} 45^{\prime \prime} \mathrm{N}, 47^{\circ}\right.$ $\left.53^{-} 41^{\prime \prime} \mathrm{E}\right)$

\subsection{Sand fly and Rodent Collection and Identifica- tion}

In this study sand flies were collected from indoors and outdoors using 150 sticky traps and the rodents using Sherman live traps from outdoor areas from May 2011-October 2011, in 3 attempts. All the traps were set at dusk and collected at dawn, the next day. All traps containing sandflies and rodents were transferred to the Dept. of Medical Entomology and Infectious and Tropical Diseases Research Center labs of Ahvaz Jundishapur University of Medical Sciences (AJUMS) to be identified. Rodent trapping and sticky traps for sand flies were performed in a rural region near the residential part of some villages. In these regions burrowing activities of rodents were seen. However, observations showed that some of residents had CL lesions. This suggests that this region may be an appropriate place for point of epidemiologic and ecologic conditions regarding CL. Therefore, by trapping 50 sand flies and 15 rodents we did the molecular experiments.

The external morphological characteristics in males including: head, claspers and terminal abdomen segments and internal morphological characteristics in females (spermatheca) were used to identify the species of sand flies (10). Next, they were identified at the species level using keys recommended by Lewis (11), Nadim and Javadian (12) and Nadim et al. (13).The rest of the abdominal segments in females were used to detect the L. major by Nested PCR application. The rodents were anesthetized using ether and the slide smears of their ear lesions were prepared using Geimsa staining. Their skull and tooth structures were used for their identification. The Iranian rodent key of Etemad was performed to identify the rodents (14).

\subsection{Molecular Characterization}

\subsubsection{DNA Extraction}

Smears were prepared from lesions. Rodents ear DNA was extracted by using High Pure PCR Template Purification Kit (Roche, Germany), according to the manufacturer's instructions.DNA was extracted as described by Aransay et al.(15), for sand flies. Briefly, individual sand fly bodies were homogenized with a sealed Pasteur pipette in 1.5-ml tubes. One hundred fifty microliters of extraction buffer ( $1 \%$ sodium dodecyl sulfate [SDS]-25 mMNaCl-25 mM EDTA) was added, and samples were placed at $65^{\circ} \mathrm{C}$ for $30 \mathrm{~min}$. Following the addition of $100 \mathrm{ml}$ of $3 \mathrm{M}$ potassium acetate ( $\mathrm{pH}$ 7.2), the homogenates were incubated on ice for $30 \mathrm{~min}$ and then centrifuged for $15 \mathrm{~min}$ at 13,000 $3 \mathrm{~g}$. Supernatants were recovered, and DNA was precipitated with the addition of $600 \mathrm{ml}$ of $100 \%$ ethanol. DNA pellets were re-suspended in $50 \mathrm{ml}$ of 0.53 Tris-EDTA (TE) (pH 8.0). Five-microliter portions of these DNA extracts were used for PCR amplification.

\subsubsection{Amplification of Kinetoplastic MinicircleDNA From Sand Flies and Rodents}

For amplification of variable minicircles of Leishmania kDNA, Nested - PCR was performed according to previous studies as follows: in the first stage two external primers CSB2XF (CGAGTAGCAGAAACTCCCGTTCA) and CSB1XF (ATTTTTCGCGATTTTCGCAGAA CG) and in the second step, two internal specific primers $13 Z$ (ACTGGGGGTTGGTGTAAAATAG) and LiR (TCGCAGAACGCCCCT) were used $(15,16)$. Ready to use Bioneer, Accupower PCR premix, 96 tubes, $0.2 \mathrm{ml}$ and $20 \mu \mathrm{lrxn}$ (Bioneer Co., Korea) were used for the PCR reaction. DNA was amplified using thermal cycler (Eppendorf AG 22331, Hamburg, Germany) under the following conditions: $5 \mathrm{~min}$ at $94^{\circ} \mathrm{C}$ followed by 35 cycles of $30 \mathrm{sec}$ at $94^{\circ} \mathrm{C}, 60 \mathrm{sec}$ at $55^{\circ} \mathrm{C}, 90 \mathrm{sec}$ at $72^{\circ} \mathrm{C}$ and a final elongation at $72^{\circ} \mathrm{C}$ for $10 \mathrm{~min}$. For each sample, one positive control and one negative control were included. Two microliters of the first-round product was used as a template for the second round in a total volume of $20 \mu \mathrm{l}$ under the same conditions as those for the first round, except with primers LiR and 13Z.The PCR products were visualized by $1.5 \%$ agarose gel electrophoresis, using a 100bp DNA ladder marker at $260 \mathrm{~nm}$. Results were compared with standard band markers of L. major (MHOM/IR/75/ER) providing fragment of $560 \mathrm{bp}$ (17). 


\section{Results}

Totally 50 sandflies were trapped during this study. All of them were identified as P. papatasi, 27 females and 23 males, from outdoor places ( 15 females and 8 males) and indoor places ( 12 females and 15 males). Totally 15 rodents including $12 \mathrm{~T}$. indica (Indian gerbil) and 3 Allactaga sp. were trapped in the current study. The Iranian rodent keys recognized 12 of the trapped rodents as $T$. indica with the following character: each of the upper incisors has one longitudinal groove in the anterior surface. The upper molars have been facilitated with fragmented grooves. The length of occipitonasal was longer than 43 $\mathrm{mm}$. The Zygomatic plate was extended forward. The tail of gerbils were darker in dorsal and ventral and lighter in the lateral sections (14).

Results of the second stage of the nested PCR technique showed that 2out of the 27 female P.papatasi (7.4\%) and1smear (ear lesion) from 12 of the T. indica (8.3\%) was positive to parasite due L. major (bands of 560bp) (Figure 2). The trapped Alactaga sp. Rodents were not infected with L. major . The positive smears of sandflies were made from indoor sandfly specimens.

\section{Figure 2. Nested-PCR(2nd Stage)}

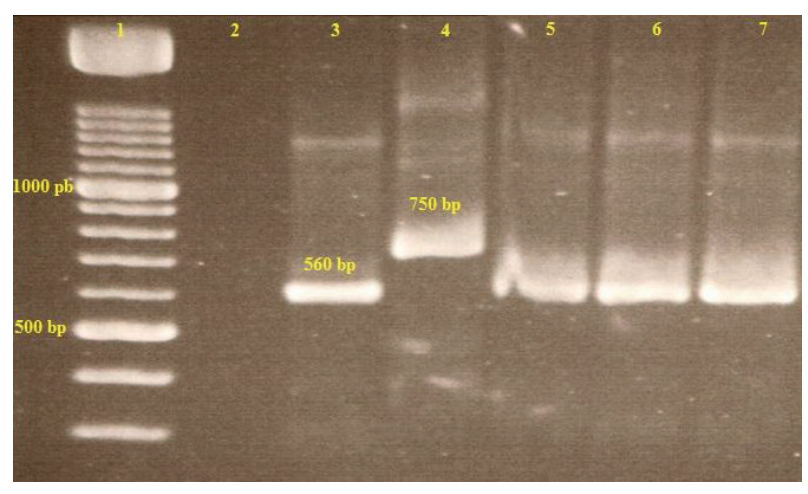

1) Marker 100 bp, 2) Negative control, 3 and 5) L. major standard, 4) L. tropica standard, 6 and 7) L. major (smear of T. indica and P. papatasi)

\section{Discussion}

A ZCL control program is based on promoting our knowledge regarding the parasite's reservoir host(s) and vectors (18). However, ZCL is endemic in Khuzestan but the majority of carried out studies have insisted on L. major of human specimens. Less studies have been performed on L. major of sandfly vectors and rodent reservoir host specimens in Khuzestan province and they belong to many years ago, when no molecular methods were used (2).P. papatasi has been confirmed as the main vector of L. major in Shoush area, north west of Khuzestan using classical entomology and parasitological methods (19).

There is a similar trend for T. indicaas reservoir of L. major in Khuzestan. All of the recent molecular Leishmania studies in Khuzestan are also related to human specimens. The current study is the first molecular report which describes naturally infected P. papatasi and T. Indica with $L$. major at the same time in the Roffaye region in the west of Khuzestan. The results of this study showed that a Nested-PCR as a sensitive and high-resolution method could detect L. major in both sand fly insects and gerbil rodents as vectors and reservoirs of the protozoan, respectively, from a region with a high rate of ZCL among the rural residents.

Results of the current study are consistent with the results of Azizi et al. (20) and Rassi et al. (21) regarding the same bands of L. major (560 bp) in P. salehi and P. papatasi, respectively $(20,21)$. However, same bands have been confirmed in human specimens of L. major in Khuzestan (9). The rate of $L$. major infection in the current study was $6.9 \%$ for female P. papatasi. This rate has been reported as 5.5\% in P. salehi from south of Iran (21). T. indica has been confirmed as the main reservoir of ZCL in the south west of Iran including Shoush of Khuzestan using classical parasitological methods (inoculation of scrapings from the ear of rodents in white mice and observing amastigotes in the mice) (5)

The rate of infected $T$. indica was reported as $12.5 \%$. In another study which was carried out in Ilam, T. indica was reported as the main reservoir of ZCL in Mehran with a rate of 5.5\% (5). Results of the current study in terms of trapped T. indica showed that $8.3 \%$ of the rodents were infected with L. major as the first report in Khuzestan by a molecular method. This rate was recorded as $11.8 \%$ among trapped Gerbillusnanus, using PCR method (22) and 3.7\% of trapped $T$. indica using classic parasitological methods in south-east of Iran (23).

According to the results of the current study, where molecular methods detected L. major in both P. papatasi and T. indica from same area and molecular reports regarding L. major in CL human specimens of different parts of west Khuzestan and similar results from classical parasitological methods, it can be concluded that P. papatasi and T. indica should be considered as a vector and reservoir of ZCL in Roffayeh. Consequently, this area could be considered as the focus of ZCL, which needs to be treated by control methods of ZCL removal.

\section{Acknowledgements}

We take this chance to thank both Infectious and Tropical Diseases Research centre, Ahvaz Jundishapur University of Medical Sciences, Ahvaz, Iran and Ahvaz Razi Vaccine and Serum Research Institute, Ahvaz, Iran for their financial support and Ali Dianat Student of Dept. Medical Entomology, Ahvaz Jundishapur University Medical Sciences Ahvaz, Iran for the collection of sandflies and rodents.

\section{Authors' Contribution}

Babak Vazirianzadeh (All parts of the research), Jasem 
Saki (PCR performance and revising the text), Elham Jahanifard (collection and identification of sandflies), Mehdi Zarean (PCR performance), Kamyar Amraee (collection and identification of the rodents), Sharokh| Navid pour (collection of sandflies and co operation in revising the text).

\section{Financial Disclosure}

There is no conflict Interest over this research study.

\section{Funding/Support}

Infectious and Tropical Diseases Research Centre, Ahvaz Jundishapur University of Medical Sciences, Ahvaz, Iran and Ahvaz Razi Vaccine and Serum Research Institute, Ahvaz, Iran.

\section{References}

1. Mahmoodi MR, Mohajery M, Tavakkol Afshari J, Taghae Shakeri M, Yazdan Panah MJ, Berenji F, et al. Molecular identification of Leishmania species causing cutaneous leishmaniasis in Mashhad, Iran. Jundishapur J Microbiol. 2011;3(4):195-200.

2. Yaghoobi-Ershadi M. Phlebotomine Sand Flies (Diptera: Psychodidae) in Iran and their Role on Leishmania Transmission. $J$ Arthropod Borne Dis. 2012;6(1):1-17.

3. Kakarsulemankhel JK. Taxonomic review of sand flies of the subgenus Paraphlebotomus Theodor (Diptera: Psychodidae). Pak Entomol. 2010;32(2):125-147.

4. Lane RP, Al-Taqi M. Sandflies (Diptera: Phlebotominae) and leishmaniasis in Kuwait. Bull entomol res. 1983;73(4):633-644.

5. Javdian E, Dehestani M, Nadim A, Rassi Y, Tahvildar Bidruni GH, Seyedi Rashti MA, et al. Confirmation of Tatera indica (Rodentia: Gerbillidae) as the main reservoir host of zoonotic cutaneous leishmaniasis in the west of Iran. Iranian J Publ Health. 1998;27(12):55-60.

6. Saki J, Meamar A, Oormazdi H, Akhlaghi L, Maraghi S, Mohebali M, et al. Mini-exon genotyping of leishmania species in khuzestan province, southwest iran. Iran J Parasitol. 2010;5(1):25-34.

7. Saki J, Khademvatan S. A molecular study on cutaneous leishmaniasis lesions in Khuzestan province (South west of Iran). Jundishapur J Microbiol. 2012;4(4):283-8.

8. Ghasemian M, Maraghi S, Samarbafzadeh AR, Jelowdar A, Kalantari M. The PCR-based detection and identification of the parasites causing human cutaneous leishmaniasis in the Iranian city of Ahvaz. Ann Trop Med Parasitol. 2011;105(3):209-15.

9. Maraghi S, Samarbaf Zadeh A, Sarlak AA, Ghasemian M, Vazirianzadeh B. Identification of Cutaneous Leishmaniasis Agents by Nested Po-lymerase Chain Reaction (Nested-PCR) in Shush City,
Khuzestan Province, Iran. Iran J Parasitol. 2007;2(3):13-5.

10. Theodor O, Mesghali A. On the Phlebotominae of Iran. J Med Entomol.1964;1:285-300.

11. Lewis DJ. A taxonomic review of the genus Phlebotomus (Diptera, Psychodidae). British Museum (Natural History);1982.

12. Nadim A, Javadian E. Key for species identification of sandflies (Phlebotominae; Diptera) of Iran. Iranian J Publ Health. 1976;5(1):35-44.

13. Nadim A, Javadian E, Seyedi-Rashti MA, Ardehali S, Rezaei R, Nadim A. Epidemiology of leishmaniasis in Iran. In: Nadim A, Javadian E, Seyedi-Rashti MA, Ardehali S, Rezaei R, Nadim A, editors. Leishmania parasite and leishmaniasis.Tehran University Publishing Center; 1994. p.179-208.

14. Etemad E. Rodents and identification key of them. 1. Tehran National Association of Natural Source Protection and Human Environment; 1979.

15. Aransay AM, Scoulica E, Tselentis Y. Detection and identification of Leishmania DNA within naturally infected sand flies by seminested PCR on minicircle kinetoplastic DNA. Appl Environ Microbiol. 2000;66(5):1933-8.

16. Noyes HA, Reyburn H, Bailey JW, Smith D. A nested-PCR-based schizodeme method for identifying Leishmania kinetoplast minicircle classes directly from clinical samples and its application to the study of the epidemiology of Leishmania tropica in Pakistan. J Clin Microbiol.1998;36(10):2877-2881.

17. Sharifi F, Sharifi I, Zarean M, Parizi MH, Aflatoonian M, Harandi MF, et al. Spatial distribution and molecular identification of leishmania species from endemic foci of South-eastern iran. Iran J Parasitol. 2012;7(1):45-52.

18. Yaghoobi-Ershadi M, Hakimiparizi M, Zahraei-Ramazani A, Abdoli H, Akhavan A, Aghasi M, et al. Sand fly Surveillance within an Emerging Epidemic Focus of Cutaneous Leishmaniasis in Southeastern Iran. Iran J Arthropod Borne Dis. 2010;4(1):17-23.

19. Javadian E, Nadim A. Studies on cutaneous leishmaniasis in Khuzestan, Iran. Part II. The status of sandflies. Bull Soc Pathol Exot Filiales. 1975;68(5):467-71.

20. Azizi K, Fakoorziba MR, Jalali M, Moemenbellah-Fard MD. First molecular detection of Leishmania major within naturally infected Phlebotomus salehi from a zoonotic cutaneous leishmaniasis focus in southern Iran. Trop Biomed. 2012;29(1):1-8.

21. Rassi Y, Abai MR, Javadian E, Rafizadeh S, Imamian H, Mohebali $\mathrm{M}$, et al. Molecular data on vectors and reservoir hosts of zoonotic cutaneous leishmaniasis in central Iran. Bull Soc Pathol Exot. 2008;101(5):425-8.

22. Azizi K, Moemenbellah-Fard MD, Kalantari M, Fakoorziba MR Molecular detection of Leishmania major kDNA from wild rodents in a new focus of zoonotic cutaneous leishmaniasis in an Oriental region of Iran. Vector Borne Zoonotic Dis. 2012;12(10):84450.

23. Kassiri H, Javadian E, Abdigoudarzi M. Natural Leishmania Infection in Meriones hurrianae and Tatera indica (Rodentia: Cricetidae: Gerbillinae) in Sistan-Baluchistan Province, South-Eastern of Iran. Adv Stud Biol 2011;3(6):247-256. 